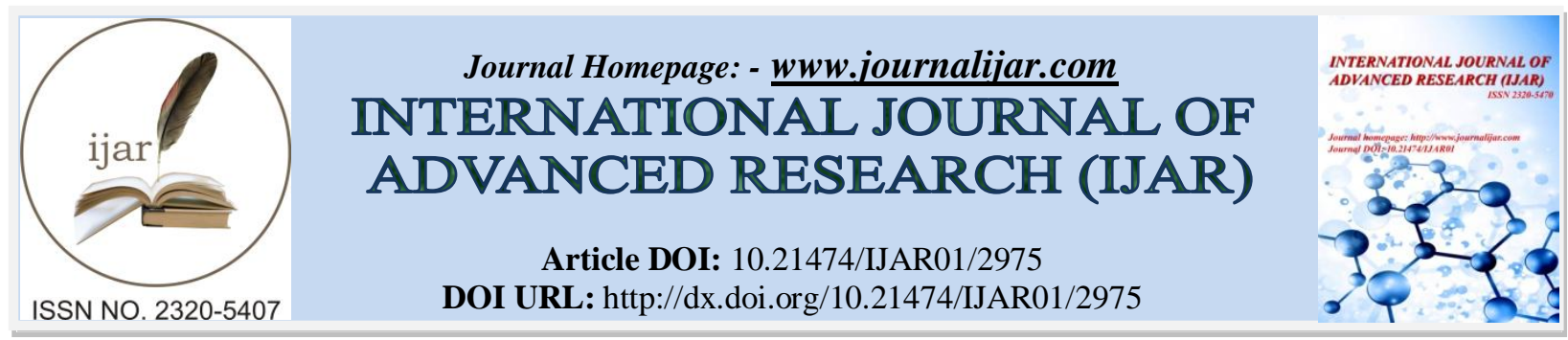

RESEARCH ARTICLE

\title{
NEW IDENTIFYING SECURITY FEATURES OF INDIAN CURRENCIES
}

\author{
Manju Sahu and Moumita Sinha*. \\ Assistant Professor, Department of Forensic Science, Guru Ghasidas University, Raipur (C.G.).
}

\section{Manuscript Info}

Manuscript History

Received: 26 November 2016

Final Accepted: 27 December 2016

Published: January 2017

Key words:-

currency forensics, currency security,

banknote recognition, counterfeit

currency, VSC

\begin{abstract}
By its definition, "currency" alludes to a concurred medium for trade, a country's cash is the formal medium authorized by the chose representing element. All through history, guarantors have confronted one basic danger: falsifying. Regardless of innovative progressions, conquering fake creation remains a far off future. Logical assurance of legitimacy requires a profound comprehension of the crude materials, assembling forms included and the security forms applied. Objective of this paper is to make layman's familiar with the security features added to new 2000 and 500 currency notes so that they can differentiate between forged and genuine currency notes with simple examinations.
\end{abstract}

Copy Right, IJAR, 2016,. All rights reserved.

\section{Introduction:-}

To fake intends to wrongfully make an impersonation of something with the purpose to exploit the prevalent estimation of the imitated item. Fake cash alludes to money that nearly look like the first money of a nation yet that is delivered without the legitimate authorize of the administration. Fake cash is unsafe to a country. Fake notes increment cash dissemination, conceivably prompting to swelling (1-3). Likewise, the general trust in the cash diminishes. Moreover, people who honestly secure fake cash are deceived. There are generally no administration approaches to repay them for fake notes that are seized. Then again, the people who deliver fake money can make huge benefits and back a variety of exercises, large portions of which may be against the national premium. Fakes are made in an assortment of ways-The least demanding and most regular path is to utilize a high determination scanner to catch both sides of a certified money note. The filtered pictures are then printed utilizing a shading inkjet or laser printer. This strategy functions admirably for little group takes note of that are normally not examined. Be that as it may, it is anything but difficult to recognize such fake notes in view of the nature of the paper that is utilized (4). A more refined strategy begins with a low group note, blanches or washes out the ink and after that prints a higher esteem note. Nonetheless, the generation of an excellent fake requires a substance to utilize a similar crude materials and printing process that are utilized to create honest to goodness cash. This out of the span of individual forgers because of the high cost and trouble in delivering the crude materials and types of gear. Security elements are frequently implanted in money notes to recognize veritable notes. Regular security highlights incorporate watermarks, security strings, inert pictures, smaller scale lettering, intaglio (raised print), optically factor ink and fluorescence (5). Notwithstanding confirming that a money note is real, the security highlights dissuade forging. Duplicating the security highlights expands the cost of forging, making it less productive. This paper concentrates on fantastic fake Indian fake notes. While the effective distinguishing proof of such notes more often than not requires master examination, it is a generally straightforward issue on the grounds that exclusive the honesty of the security highlights must be researched. A more troublesome assignment is to recognize the wellspring of a fake note. This requires point by point examination by legal researchers utilizing costly instruments. Likewise, no programmed technique exists to interface a fake note to source press. Indian money notes are printed at 
committed government printing presses. The quantity of presses is restricted and extraordinary care is taken amid its printing. This paper essentially concentrates on the remarkable elements of money notes made for security reason and can help the layman to comprehend that straightforward mindfulness identified with notable elements of new monetary forms can help them to anticipate themselves of being casualties to forgers. In a noteworthy stride to check undeclared dark cash, the Government of India on the 8 November, 2016 declared demonetization of Rs. 500 and 1000 banknotes with impact from that day's midnight making these notes invalid. The Indian 2000 rupee banknote is of the most noteworthy division of Indian money. It was initially presented by Reserve Bank of India in November 2016. Indeed, even as Rs 2,000 notes printed by the Reserve Bank of India (RBI) are yet to achieve banks all over the place, a fake note of this section has surfaced many places of India. The fake notes found in different parts of the nation were photocopied adaptations, this note had highlights including the Gandhi watermark alongside the national image and a fake security string (6-7). Below some of the salient features of $\square 2000$ and $\square 500$ are mentioned which can help common people to differentiate between fake and genuine currencies.

\section{Material and Methods:-}

Present study has been undertaken to study spectral examination of various security features in New Indian paper currency prescribed by Reserve Bank of India. New Currency note of Mahatma Gandhi series of denomination 2000 and 500 was selected for present study. The absorption spectral characteristics were examined by video spectral comparator (model 40). Various types of features were examined on paper currency note using different lights ranges (UV-365,312, 254nm, Ultra Violet transmitted 365nm), by using different magnification ranges and spot light, various filters. In these ranges various security features were analyzed.

\section{1) New Security Features of Rs. 2000 notes (Figure1a and b):}

The Reserve Bank of India will issue $\square 2,000$ denomination banknotes in the Mahatma Gandhi (New) Series, without the inset letter.

\section{Front side features:-}

1. Size: $66 \mathrm{~mm} \times 166 \mathrm{~mm}$, smaller than the old notes,

2. Colour: Rs 2,000 note's paper will be available in a magenta base color.

3. Registered denominational numeral 2000 is seen.

4. Latent image with denominational numeral 2000 is observed.

5. Denominational numeral २००० in Devnagari is observed.

6. At the centre Portrait of Mahatma Gandhi is seen.

7. On the left side of the banknote Micro letters 'RBI' and '2000' observed.

8. On banknotes windowed security threads with inscriptions 'भारत, RBI and 2000 with color shift are observed. Thread Color changes from green to blue when the note is tilted.

9. Towards right side guarantee clause, governor's signature with promise clause and RBI emblem.

10. Watermarks of electrotype and Mahatma Gandhi portrait (2000).

11. On the top left side and bottom right side number panel with numerals growing from small to big.

12. On bottom right denominational numeral with Rupee Symbol, $\square 2000$ in changing color ink (green to blue).

13. On left and right side raised print of seven angular bleed lines are present.

14. Emblem of Ashoka Pillar on the right.

\section{Reverse side features:-}

15. On the left of the note year of printing is seen.

16. Swachh Bharat logo with slogan is added as a new feature.

17. Language panel towards the centre on the reverse side.

18. Motif of Mangalyaan is added as a new feature.

The notes are also meant to have some special features that have been designed keeping the visually-impaired individuals in mind.

Intaglio or raised printings of Mahatma Gandhi portrait, Ashoka Pillar emblem, bleed lines and identity mark.

19. Raised print of $\square 2000$ horizontal rectangle form observed on the right.

20. On left and right side seven angular raised print bleed lines observed. 


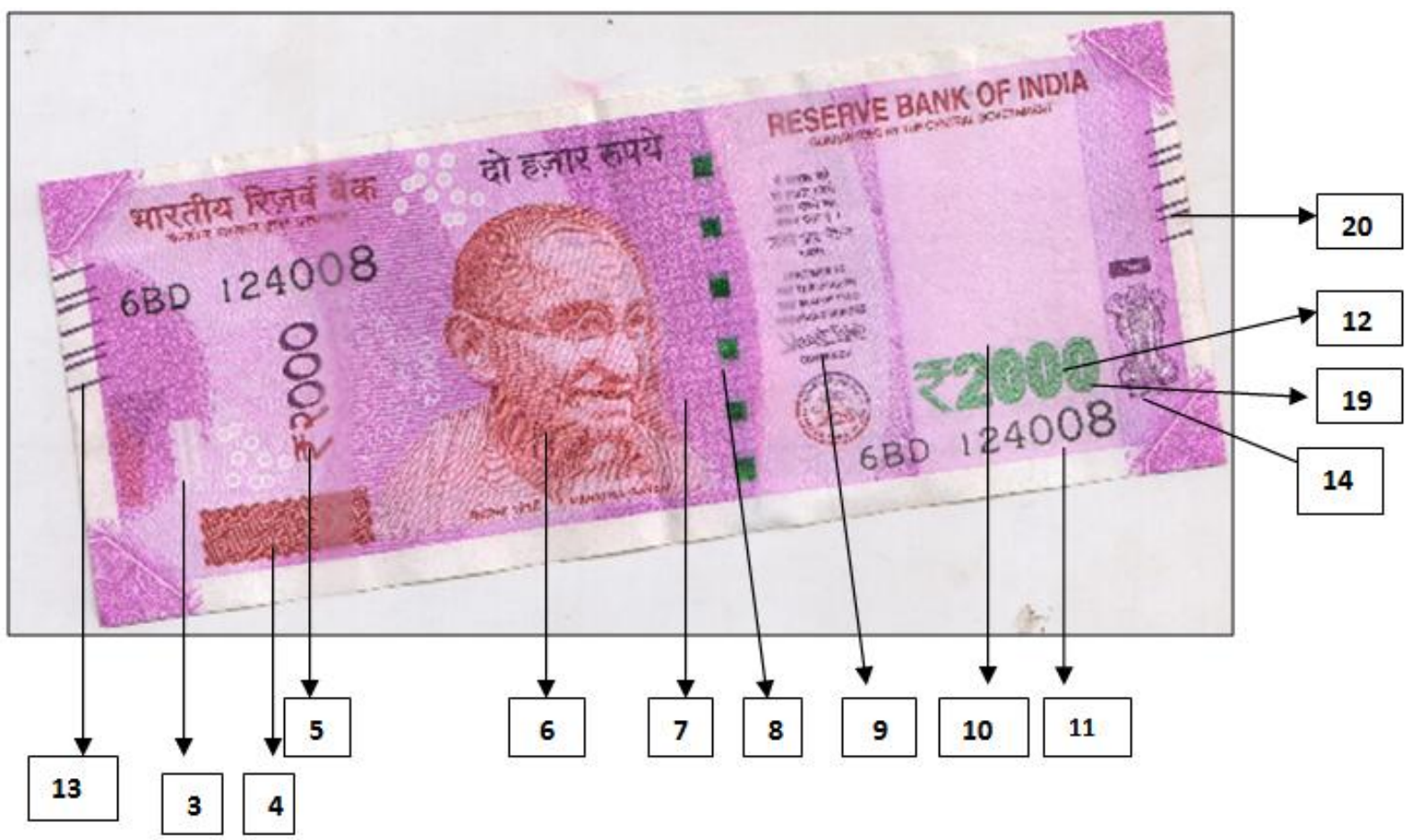

Figure1a:- Showing identifying features of Rs. 2000 currency of front side under VSC.

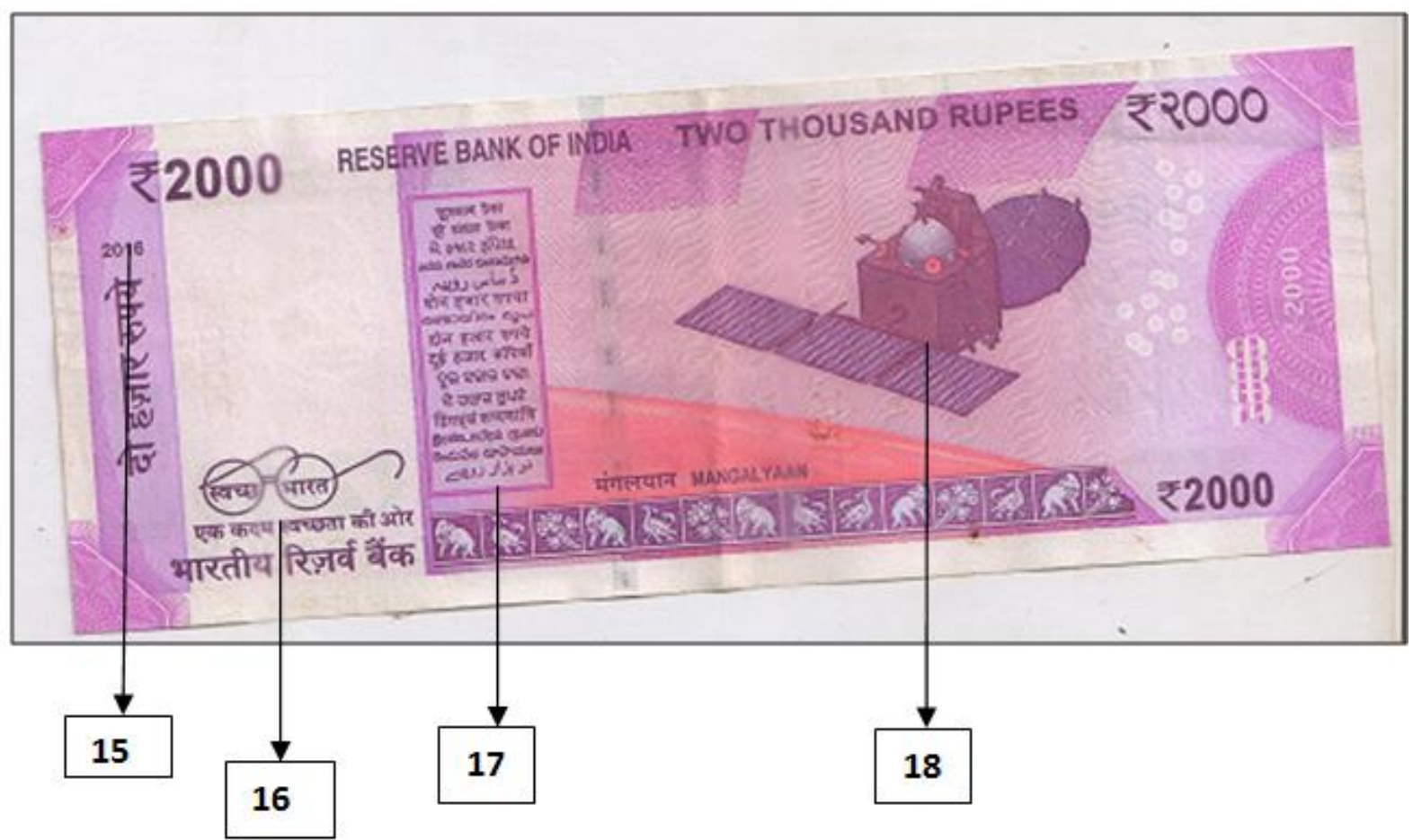

Figure1b:- Showing identifying features of Rs. 2000 currency of reverse side under VSC.

New Security Features of Rs. 500 notes (Figure2a and b):-

The RBI will issue $\square 500$ denomination banknotes in Mahatma Gandhi (New) Series with inset letter 'E' in both the number panels.

1. Size: The new Rs 500 note at $63 \mathrm{~mm} \times 150 \mathrm{~mm}$ is smaller in size than previous one. 
2. Color: Color is stone grey with Red Fort and Mahatma Gandhi's image on each side.

3. Registered denominational numeral 500 is seen.

4. The denomination numeral in latent image.

5. Denomination numeral in Devnagari script.

6. Mahatma Gandhi's portrait orientation changed.

7. When note is tilted windowed security thread changes from green to blue.

8. RBI emblem, Guarantee clause, Governor's signature shifted towards right.

9. Portrait of Mahatma Gandhi and electrotype watermarks.

10. Number panel with numerals growing from small to big on top left and bottom right sides.

11. On the bottom right denomination numerals with rupee symbol in colour changing ink from green to right.

12. Ashoka pillar emblem can be seen on the right side.

For Visually Impaired Mahatma Gandhi portrait, Ashoka Pillar emblem and identification mark in raised print.

13. 500 in raised print on the right with circle.

14. On the left and right five bleed lines in raised print.

\section{Reverse Side}

15. Printed year on the left side.

16. Logo of Swatch Bharat with slogan.

17. Language panel towards centre.

18. Red fort image with Indian flag.

19. Numeral of denomination in Devanagari on the right.

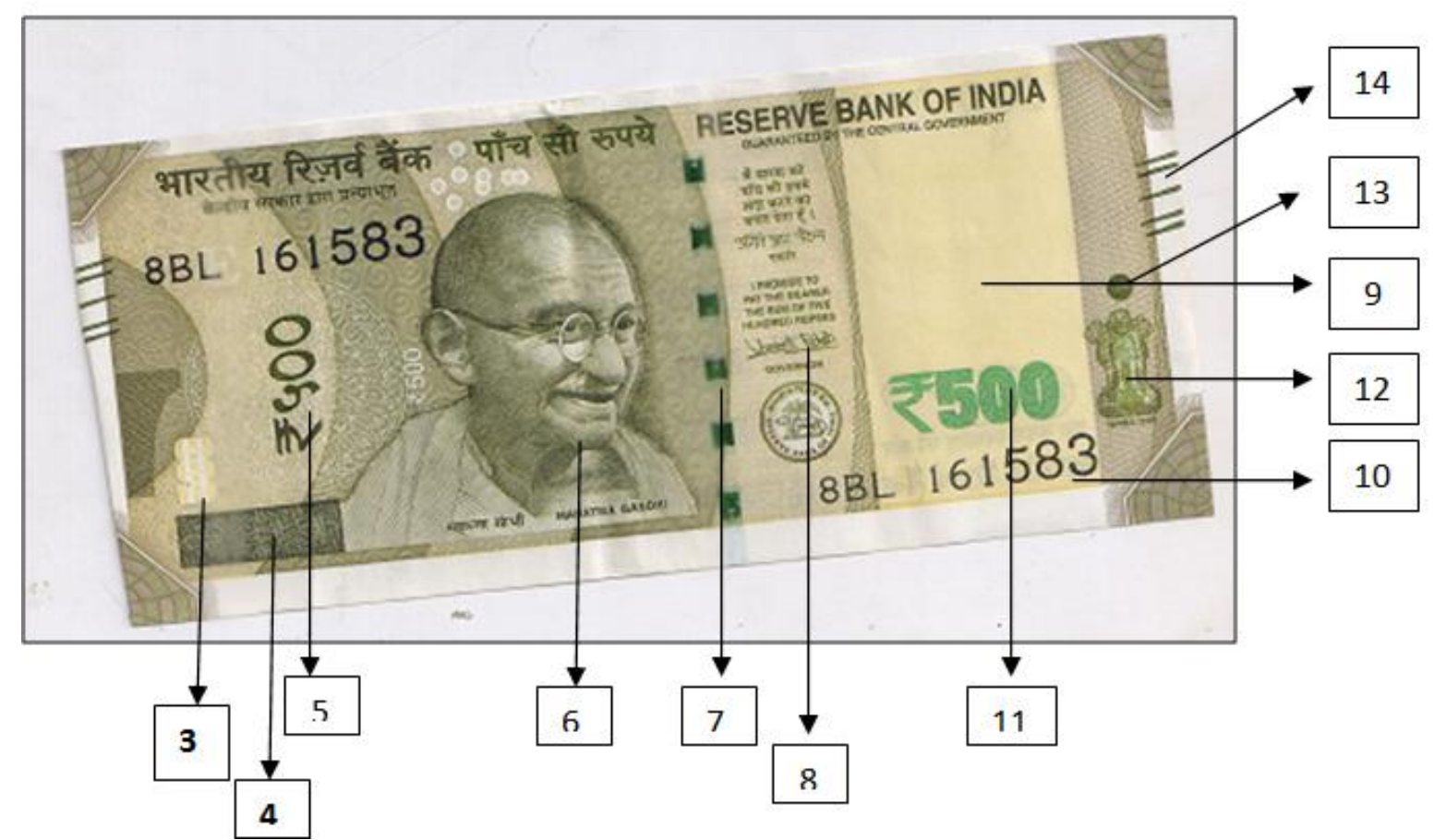

Figure 2a:- Showing identifying 


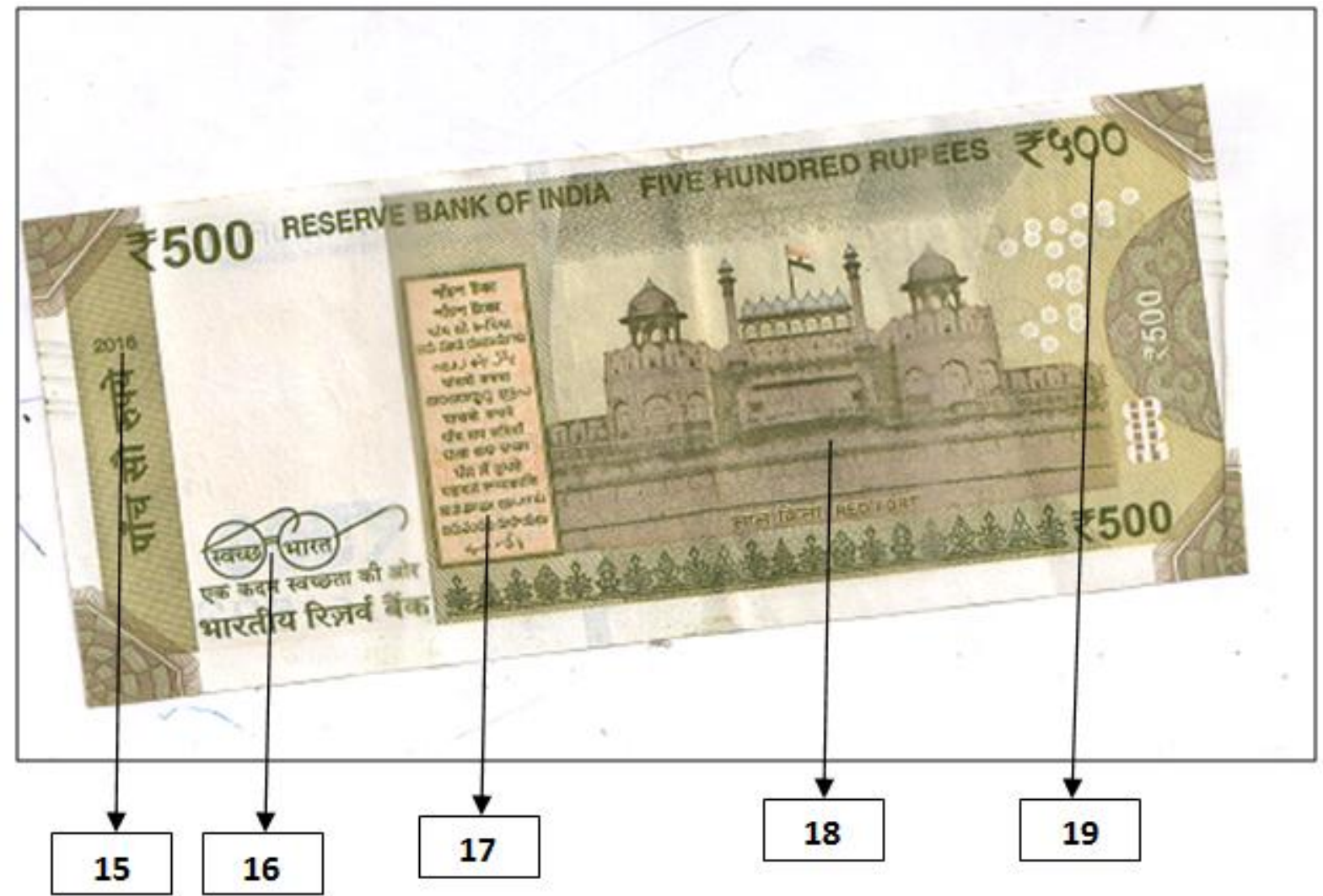

Figure 2b:- Showing identifying features of Rs. 500 currency of front side under VSC.

\section{Conclusion:-}

Exhibit study was done to dissect the different real elements of Indian cash of division 2000 and 500 utilizing Video Spectral comparator-40 (Freeman-Foster) to concentrate different certifiable components according to the rules of RBI. Exhibit study can be exceptionally useful in recognizing bona fide and fake Indian cash notes. The technique received is very advantageous and bona fide which can be increasingly embraced by bank authorities, security offices, cash exchangers and legal specialists. The methodology is exceedingly fitting, speedy and easy to use without much of a stretch be dissected and easily explained in report.

\section{References:-}

1. Sharma B. K. "Counterfeiting of Indian Currency", CBI bulletin vol. VIII NO.11 NOV., pp. $4-7$ (Special issue on counterfeiting of currency), 2000.

2. Oliver J., Chen J. "Use of Signature Analysis to Discriminate Digital Printing Technologies", in Proc. of the Int. Conf. on Digital Printing Technologies, pp. 218-222, San Diego, California,2002.

3. Mittal S. C., Arora N. "Forgery of rupees Five Hundred Denomination Notes-Methods of Detection", CBI Bulletin, II, pp.4-20, 2003.

4. Mittal S. C. "Recent trends in forgery of Rs. 500 denomination Bank Notes of Mahatma Gandhi Series \& their detection", CBI Bulletin, 12, pp. 8, 2004.

5. Mittal S. C., Arora N. "Recent Trends in the Counterfeiting of Rupees 1000 Denomination Currency NotesMethods of Detection", NCRB Gazette, XVII (3), pp. 29-47, 2005.

6. Li C. K., Chan W.C., Cheng Y. S., Leung S. C. "The Differentiation of Color Laser Printers,"Journal of the American Society of Questioned Document Examiners, Vol.7 (2), pp. 105-109,2004.

7. Pal A., H. K. Pratihari. "Spectral Studies on Original and Fake Rupees 1000 Denomination Note" ISSN09743111 volume 4(2), 91-96, 2012. 